Physics, Chemistry, and Dynamics of Interplanetary Dust

ASP Conference Series, Vol. 104, 1996

Bo A. S. Gustafson and Martha S. Hanner (eds.)

\title{
Space Debris
}

D.J. Kessler

NASA Johnson Space Center, C23, Houston, TX 77058

\author{
P.D. Anz-Meador
}

Viking Science \& Technology, Inc., 16821 Buccaneer Lane, Suite 106, Houston, TX 77058

\author{
M.J. Matney
}

Lockheed Martin Engineering and Science Services, NASA Johnson Space Center, C23, Houston, TX 77058

\begin{abstract}
Man-made, artificial space debris is of interest to the study of interplanetary dust for two reasons: (1) In many regions of Earth orbital space, the space debris flux is larger than the natural meteoroid flux, complicating the study of interplanetary dust, and (2) models and experiments developed to understand space debris may have application to the study of interplanetary dust. The purpose of this paper is to summarize the space debris environment as it is understood today by characterizing the models used to predict the space debris environment and describing the measurements to test the model predictions.

Within the last 5 years, the space debris environment has been measured by a number of experiments. These experiments have revealed significant sources of debris in addition to the assumed major source of satellite explosions. Understanding these sources has required the development of more complex models and additional insight into the design and operation of spacecraft. Increased awareness of space debris issues at an international level has led to measures that have reduced the rate of growth in the environment. However, the number of new debris sources discovered seems to be proportional to the number of new measurements of the environment.
\end{abstract}




\section{Introduction}

A single spacecraft orbiting below $2000 \mathrm{~km}$ altitude is capable of producing a manmade orbital debris hazard which exceeds the natural interplanetary meteoroid hazard in low Earth orbit. Recause of the high inclinations that most spacecraft are launched into, the average collision velocity between objects in this region is about $10 \mathrm{~km} / \mathrm{sec}$ (Kessler 1994). Consequently, it was inescapable that orbital debris would become an environmental issue requiring models and measurements to understand this new environment. The models and measurements developed are identical in many respects to the models and measurements used to understand the interplanetary meteoroid environment. Both the meteoroid and orbital debris investigators can, and have, benefited by sharing data and modeling techniques. The purpose of this paper is to summarize current orbital debris models and measurements that may be of interest to the interplanetary meteoroid investigators.

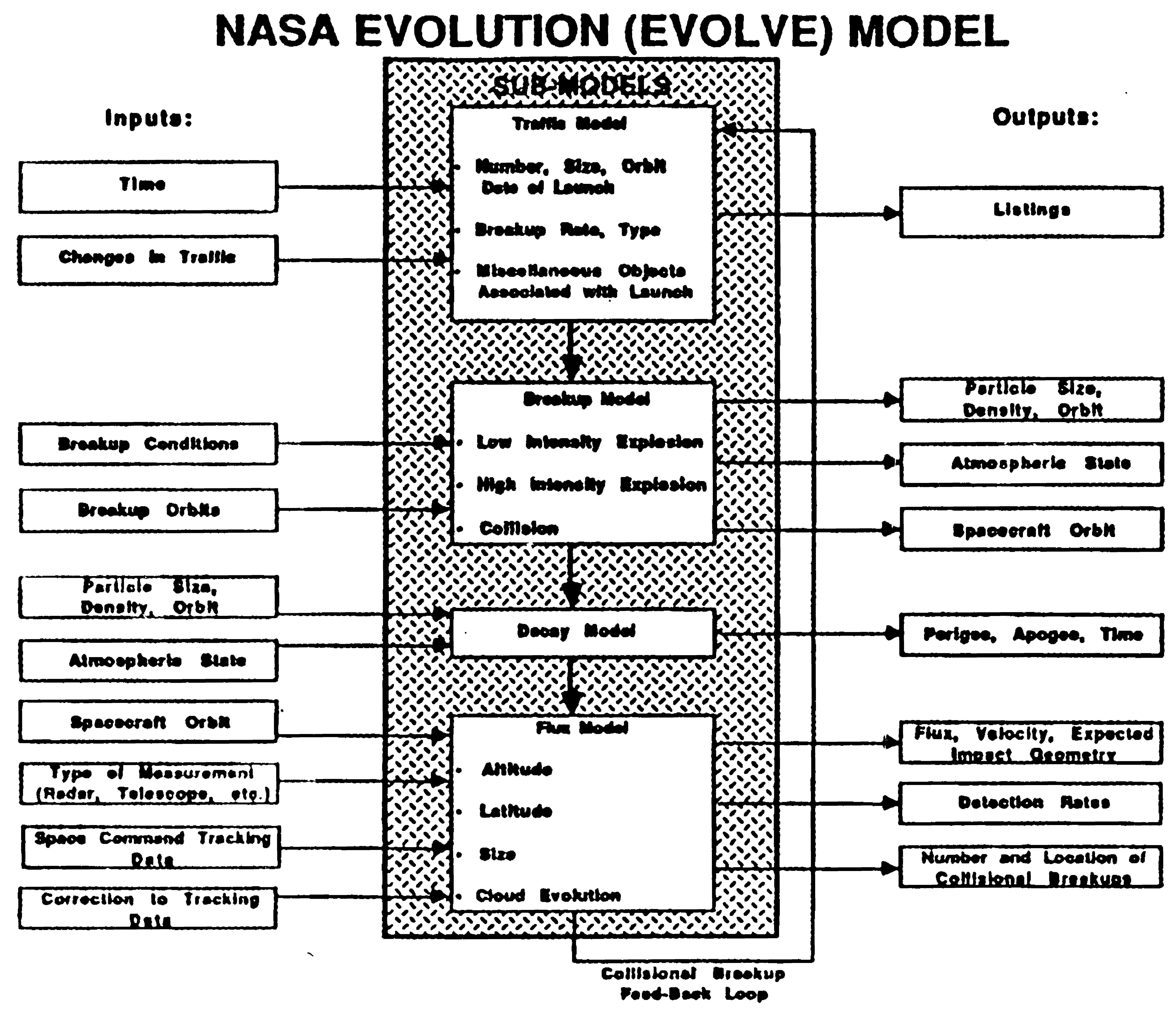

Figure 1: Structure of the NASA Evolution (EVOLVE) model 


\section{Orbital Debris Models}

Fundamentally, there are three types of orbital debris models. The most complex and complete model is the "evolutionary" model (Reynolds, et al. 1995, Klinkrad, et al. 1995, and Rossi, et al. 1994). Figure 1 illustrates the logic flow of the NASA model "EVOLVE". The NASA model assumes rates of launch and orbital characteristics of objects placed in Earth orbit. Satellite breakup models are required to predict the new orbits of fragments resulting from an explosion in orbit. Over 100 explosions are known to have occurred in low Earth orbit (Gleghorn, et al. 1995). An atmospheric density model and solar activity predictions are used to decay objects and predict their orbital perigee and apogee as a function of time. Orbital elements are then converted to an average collision rate using equations developed by Kessler (1981) to obtain flux as a function of debris size. These calculated collision rates are then used to predict the rate of satellite breakups due to collisions with other debris and the resulting rates are fed back into the traffic model.

Just as with natural objects, the number of man-made objects increases rapidly with decreasing size. Consequently, if one attempted to maintain the identity of all individual objects, computation time would become prohibitive large as one tried to describe the environment for smaller debris. To keep the computation time manageable, below a certain size, random samples are maintained and weighted according to the fraction of the total population that they represent. As a result, the computer run times for EVOLVE require a few hours to predict a few hundred years into the future.

However, computer runs of a few hours are much too long for many applications. In addition, because the evolutionary model must assume sources in order to predict the environment, many times the resulting predictions are not consistent with measurements because of either unknown sources, uncertainties in satellite breakup models or uncertainty in the effects of atmospheric drag. The "engineering" model attempts to combine the results of the evolutionary models and measurements into a simple model that can be used for planning experiments and to design protection for spacecraft. An example of a simple engineering model that describes the meteoroid environment is the set of equations describing the meteoroid flux at 1 A.U. given by Grün, et al. (1985). A more complex meteoroid engineering model which predicts the flux and velocity at various distances from the sun is given by Divine (1993).

Until recently, there was sufficient uncertainty in the orbital debris environment that only a simple engineering model was justified, as given by Kessler, et al. (1989). This simple model was not much more complex than the Grün meteoroid model. However, with much higher quality measurements by the LDEF satellite and by the Haystack radar, knowledge of the orbital debris environment has grown to the point where the 1989 engineering model is inadequate. Consequently, NASA is currently developing a more complex, computer-based orbital debris engineering model. This model assumes orbit distributions similar to those in Divine's approach; however, it uses the equations developed by Kessler (1981) to convert these distributions to flux and velocity. Matney and Kessler (1995) have shown that using the Kessler equations 
in this manner is identical to Divine's approach, if the orbit distributions are defined in the same way. This new engineering model is near completion and has a computer run time of less than 1 second.

A third type of model is the "special purpose model". This type of model is used to address some special problems that may be outside the capabilities of either of the other two models. For example, the effects of solar radiation pressure would require excessive computer time to accurately include in the EVOLVE model and it is also not important for most sizes of interest. However, by developing a model which describes orbital parameter changes on a single particle as a result of solar radiation pressure and atmospheric drag, these effects can be quantified and, if needed, approximated in either the EVOLVE or Engineering model. As a result of such a model, it has been concluded that solar radiation pressure is increasingly important in describing the altitude distribution of orbital debris in low Earth orbit with decreasing size below 100 microns.

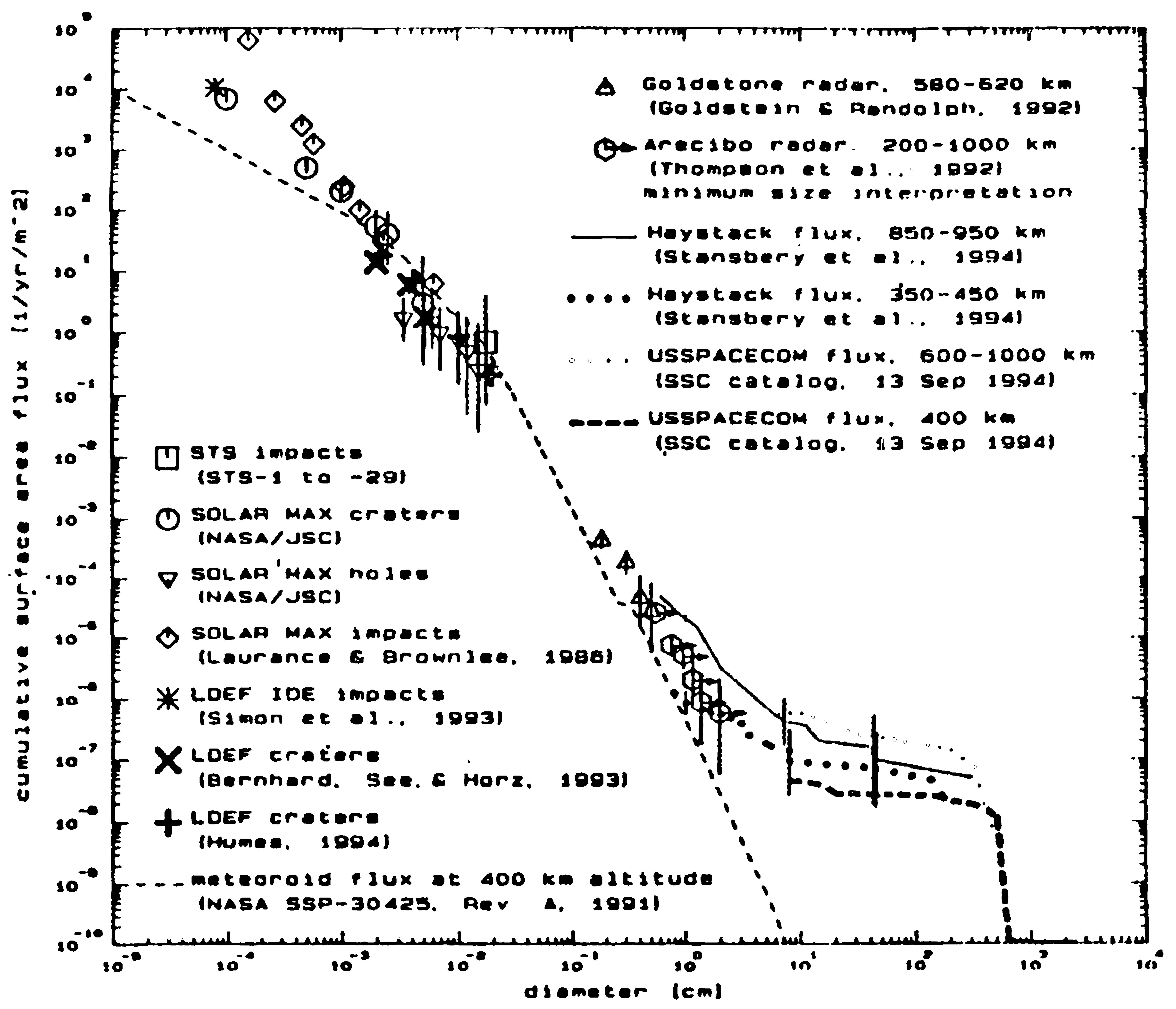

Figure 2: Orbital debris measurerrents compared to meteoroid environment 


\section{Measurements}

Until the 1980's, all orbital debris measurements were conducted by the US Space Command and no recognized measurements of the population existed for orbital debris sizes smaller than about $10 \mathrm{~cm}$. Today, the populations in low Earth orbit for nearly all size ranges have been measured by various techniques. Figure 2 gives a summary of the more recent, major measurements. As predicted by early models, the debris flux exceeds the natural meteoroid flux over most of the size region. Two of the measurements of smaller debris are particularly important because of the large amount of information that has been obtained by these measurements. These are the LDEF and Haystack radar measurements.

On LDEF, there were a number of experiments that measured the meteoroid and orbital debris populations, but three of them especially introduced important new data that was useful toward understanding the orbital debris population: (1) The Meteoroid and Space Debris Impact Experiment (Humes 1993), (2) The Chemistry of Meteoroids Experiment (CME) (Hörz, et al. 1993), (3) The Interplanetary Dust Experiment (IDE) (Oliver, et al. 1993). Of these three experiments, only Humes' experiment originally stated as an objective to measure space debris. The large area of the experiment provided adequate statistics to use the directional properties of orbital debris and meteoroids to help determine the relative contributions of these two components (Zhang and Kessler 1993). Only aluminum surfaces were available when the experiment was assembled, so that aluminum orbital debris impacts could not be identified by their chemistry.

The best chemistry data was obtained from the CME. Because one of the surfaces was gold and located on a trailing surface, unique chemical and orbital information on orbital debris was obtained. The chemical information indicated that by far, the most common orbital debris impactors were aluminum. For smaller sizes, this aluminum was in the form of aluminum oxide, while only pure aluminum was detected for the larger sizes (Bernhard, et al. 1995). However, the amount of manmade debris detected on this rear-facing surface was many times the amount expected. For orbital debris to impact this surface, the debris had to be low inclination, high eccentricity orbits, and few objects have been catalogued in these types of orbits. However, this is a difficult orbit for US Space Command to observe and catalogue objects. This LDEF result has put new emphasis on understanding debris in these orbits. Other debris identified on the CME included paint flecks, stainless steel, copper and silver.

An objective of the IDE was to find natural micro-meteoroid showers; instead, the experiment detected swarms of earth orbiting particles. Although this was totally unexpected, in retrospect, perhaps it should not have been surprising. Earth orbiting particles smaller than 1 micron have a very short orbital lifetime. Consequently, the ascending node of their orbit does not have time to change significantly before they enter the Earth's atmosphere. Therefore, if some objects were continuously releasing small particles (such as paint being released by the eroding action of atomic oxygen) one might expect these small particles to be in the orbital plane of the source of the particles, and appear as a swarm when LDEF crossed the orbital plane of the source. We have been trying to identify sources for individual swarms detected by the IDE; 
although we have many candidates, so far we have not been able to uniquely identify any particular object as being the necessary source.

The Haystack radar measurements represent the first effort to monitor the orbital debris population larger than about $0.5 \mathrm{~cm}$ (Stansbery, et al. 1995). The Haystack radar, located near Boston, is a high power, X-band, monopulse tracking radar with a very high sensitivity. The radar is operated by detecting objects which pass through its 0.05 degree field of view as the radar stares at some point in space. Figure 3 gives the results of over 600 hours of vertical staring observations. The data is compared with the frequency that one would expect catalogued objects to pass through the field of view, and with the Kessler, et al. 1989 model prediction. There are some clear differences with the model predictions. The most surprising difference was the peak count rate between $850 \mathrm{~km}$ and $1000 \mathrm{~km}$. All the models predicted that smaller debris would be created from explosions and would be spread out much more in altitude due to the delta-velocities imparted from the breakup events. Therefore, it was concluded that these objects in this range could not be from explosions, and that a new source was required to explain them.

The Haystack radar is also capable of measuring polarization, range rate, and direction of motion. Also, from a project to calibrate ground fragments sizes with radar return signal, the size of debris was determined. Combining all of the available data from Haystack program, it was concluded that this new source for debris had released about $30 \mathrm{~kg}$ of nearly spherical particles smaller than about $2 \mathrm{~cm}$ in diameter into near-circular orbits with altitudes between $850 \mathrm{~km}$ and $1000 \mathrm{~km}$ and orbital inclinations near 65 degrees. In addition, because the altitude distribution did not change with time due to orbital decay, the mass density of these objects was concluded to be greater than $0.5 \mathrm{~g} / \mathrm{cm}^{3}$.

By examining the orbital characteristics and engineering design of potential sources for this debris, it was concluded that the leakage of the coolant used in a class of Russian spacecraft, known as "RORSATs", was totally consistent with these findings. The coolant used is a sodium/potassium $(\mathrm{NaK})$ alloy which has a melting temperature of around -11 degrees Celsius. These finding are also consistent with some lower altitude observations by the Goldstone radar of swarms of debris which appeared to be coming from Cosmos 1900, the only RORSAT left at a lower altitude (Stansbery, et al. 1995).

If $\mathrm{NaK}$ particles smaller than 100 microns are generated, then they could be expected to have decayed to LDEF altitude and contributed to the impacts found on LDEF. Bernhard et al. (1995) reexamined LDEF craters where sodium and potassium had been detected. Previously, the presence of these elements were believe to indicate a salt contamination, and not representative of the impacting particle. Out of 1000 craters where chemical data was available, 2 of them have now been identified as containing only $\mathrm{NaK}$.

The Haystack radar has also identified another unknown source. When the radar is pointed south, so that lower inclination debris can be detected, it also detects more centimeter size debris than our models predict. This observation is consistent with the observations of LDEF of an unknown source of low inclination orbital debris. Haystack does not yet have a sufficient amount of data to determine if these objects 
are in highly elliptical orbits, but these inclinations are used to place objects into geosynchronous orbits and the spent rocket bodies are often left in their elliptical transfer orbits, so high eccentricity orbits for the debris are at least probable.

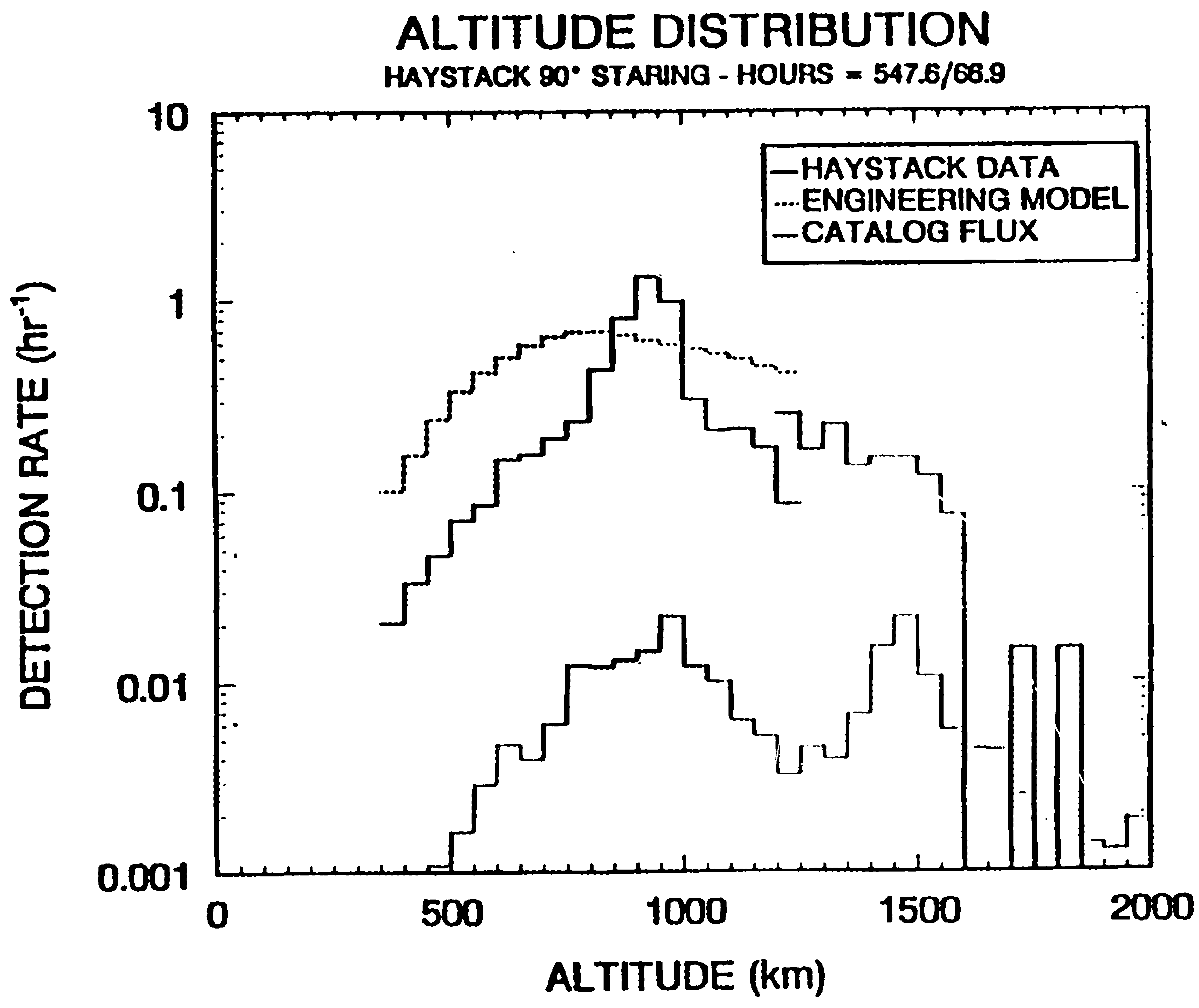

Figure 3: Haystack radar altitude distribution compared to the Engineering model. The catalog distribution represents objects larger that about $10 \mathrm{~cm}$ in diameter, and the Haystack data represents particles larger than a limiting diameter around $1 \mathrm{~cm}$. Note the conspicuous peak of small debris in the Haysatck data between 850 and $1000 \mathrm{~km}$ altitude.

\section{Conclusions}

Models have predicted that the orbital debris environment could exceed the meteoroid environment in certain regions of low Earth orbit. Observations have confirmed this prediction, and have shown that the effects are dependent on the pointing direction and size regime (Love and Brownlee 1993). However, the observations are also revealing sources of debris that were not predicted. An increased measurements program is essential towards understanding and controlling the growth in the future orbital debris 
environment. By working together, both orbital debris and meteoroid research has greatly benefited, and hopefully will continue to benefit in the future.

\section{References}

Bernhard, R. P., F. Hörz, and D. J. Kessler 1995, presented at an International Workshop on Space Debris, Moscow, Russia, October, 1995

Divine, N. 1993, JGR, 98, E9, 17,029

Grün, E., H. A. Zook, H. Fectig, and R. H. Giese 1985, Icarus, 62, 244

Gleghorn, G., J. Asay, D. Atkinson, W. Flury, N. Johnson, D. Kessler, S. Knowles, D. Rex, S. Toda, S. Veniaminov, and R Warren 1995, Orbital Debris: A Technical Assessment, National Academy of Sciences

Hörz, F. R. P Bernhard, T. H. See, D. E. Brownlee 1993, LDEF-69 Months in Space, Third Post-Retrieval Symposium, NASA CP-3275, 1, 415

Humes, D. H. 1993, LDEF-69 Months in Space, Third Post-Retrieval Symposium, NASA CP-3275, 1, 287

Kessler, D. J. 1981, Icarus, 48, 39

Kessler, D. J., R. C. Reynolds, and P. D. Anz-Meador 1989, Orbital Debris Environment for Spacecraft Designed to Operate in Low Earth Orbit, NASA TM 100-471

Kessler, D. J. 1994, The Current and Future Environment: An Overall Assessment. Preservation of Near-Earth Space for Future Generations, J. A. Simpson, Cambridge University Press, 1994, 19

Klinkrad, H., H. Sdunnus, and J. Bendisch 1995, Adv. Space Res., 16, (11) 93

Love, S. G., and D. E. Brownlee 1993, Science, 262, 550

Matney, M. J., and D. J. Kessler 1995, this volume

Oliver, J. P., S. F. Singer, J. L. Weinberg, C. G. Simon, W. J. Cooke, P. C. Kassel, W. H. Kinard, J. D. Mulholland, and J. J. Wortman 1993, LDEF-69 Months in Space, Third Post-Retrieval Symposium, NASA CP-3275, 1, 323

Reynolds, R. C. and P. Eichler 1995, Adv. Space Res., 16, (11)127

Rossi, A., A. Cordelli, P. Farinella, and L. Anselmo 1994, JGR, 99, Ell, 23,195

Stansbery, E. G., D. J. Kessler, and M. J. Matney 1995, ALAA Paper 950664

Zhang, J. and D. J. Kessler 1993, LDEF-69 Months in Space, Third PostRetrieval Symposium, NASA CP-3275, 1, 373 